\title{
La question de l'expérimentation et du naturel dans les études sur l'intelligence animale en France et aux États-Unis au début $\mathrm{du} \mathrm{xx}^{\mathrm{e}}$ siècle
}

\section{Marion Thomas}

\section{Q OpenEdition}

\section{Journals}

Édition électronique

URL : https://journals.openedition.org/cps/2738

DOI : $10.4000 /$ cps. 2738

ISSN : 2648-6334

Éditeur

Presses universitaires de Strasbourg

Édition imprimée

Date de publication : 15 décembre 2010

Pagination : 45-70

ISBN : 978-2-35410-037-5

ISSN : $1254-5740$

Référence électronique

Marion Thomas, « La question de l'expérimentation et du naturel dans les études sur l'intelligence animale en France et aux États-Unis au début du xx siècle », Les Cahiers philosophiques de Strasbourg [En ligne], 28 | 2010, mis en ligne le 15 mai 2019, consulté le 08 novembre 2022. URL : http:// journals.openedition.org/cps/2738; DOI : https://doi.org/10.4000/cps.2738

\section{(c) (i) (3) (2)}

Creative Commons - Attribution - Pas d'Utilisation Commerciale - Partage dans les Mêmes Conditions 4.0 International - CC BY-NC-SA 4.0

https://creativecommons.org/licenses/by-nc-sa/4.0/ 


\section{La question de l'expérimentation et du naturel dans les études sur l'intelligence animale en France et aux États-Unis au début du $\mathrm{XX}^{\mathrm{e}}$ siècle}

\section{Marion Thomas}

La parution en 1859 de L'Origine des espèces de Charles Darwin marque une révolution conceptuelle dans les sciences de la nature en posant l'évolution des espèces, l'homme y compris. Mais c'est surtout dans La Filiation de l'homme et la sélection liée au sexe (1871) que Darwin met en évidence des ressemblances tant anatomiques que physiologiques entre l'homme et l'animal pour prouver l'origine animale de l'homme. Il laissera à son disciple George Romanes le soin de prendre en charge les questions psychologiques, en particulier la question de l'évolution de l'esprit. Parmi les sujets de recherche qui illustrent cette question, celle de l'intelligence est centrale.

Bien sûr, Romanes n'est pas le premier à chercher à savoir si l'on peut prêter l'intelligence aux animaux. La question de l'intelligence fut un problème philosophique avant d'être un problème scientifique. Il suffit de penser aux positions antinomiques de Montaigne et de Descartes. Dans L'Apologie de Raymond Sebond (1580) Montaigne atteste que l'homme n'a pas le monopole de l'intelligence, mais qu'il partage avec les autres animaux, selon les ordres et des degrés différentes, une seule et même loi de la nature. Descartes, de son côté, ne voit dans les bêtes que des machines plus ou moins bruyantes qui, même si douées de sensibilité (elles peuvent souffrir), n'en sont pas moins privées de conscience et $a$ fortiori, d'intelligence.

Notre objectif ici est d'étudier la question de l'intelligence animale en tant que problème scientifique dans un contexte post-darwinien et d'en mesurer les enjeux épistémologiques et politiques. À travers 
la comparaison d'études réalisées dans deux contextes géographiques (américain et français), à une époque similaire (début du XXe siècle) par, respectivement le psychologue américain Robert Yerkes, le zoologiste français Louis Boutan et les psychologues français Paul Guillaume et Ignace Meyerson, nous chercherons tout d'abord à relier l'étude de l'intelligence animale à la question de la preuve expérimentale. On examinera notamment les critères d'objectivité avancés par les scientifiques pour légitimer leurs méthodologies au moment où les prérogatives du laboratoire, à savoir, le contrôle, la répétition et la quantification s'imposent au détriment de l'observation sur le terrain. Ensuite, nous chercherons à mettre en évidence l'influence de la nécessité de l'expérimentation en laboratoire dans la conception du naturel et la construction de la naturalité du comportement animal par ces mêmes scientifiques.

\section{L'étude de l'intelligence des singes par Robert Yerkes: de la station expérimentale de Californie aux tests d'intelligence de l'armée américaine et des bureaux d'immigration}

La psychologie comparée aux États-Unis, au début du XX'e siècle est marquée par la personnalité de John Watson (1878-1958). En 1909, à l'époque où il est professeur à l'université Johns Hopkins, Watson va se lier d'amitié avec une autre figure majeure de la psychologie américaine, Robert Yerkes (1876-1956) alors professeur assistant en psychologie comparée à l'université de Harvard. Dans un premier temps, la collaboration entre les deux scientifiques est fructueuse et ils ont une vision suffisamment commune de leur discipline pour fonder, en 1911, le Journal of Animal Behavior. ${ }^{1}$ Ils contribuent aussi à faire de la souris le modèle standard de laboratoire en psychologie. Cependant, leurs relations professionnelles se détériorent après l'année 1913.

Cette année-là, dans un texte devenu un manifeste, Watson proclame que la psychologie ne doit plus saisir le psychisme à travers une démarche introspective mais à travers le comportement (behavior) des corps. Autrement dit, en cherchant à définir la psychologie comme

1 Pour une étude approfondie des relations entre Yerkes et Watson, voir Radick, G. The Simian Tongue, The Long Debate about Animal Language, Chicago and London, The University of Chicago Press, 2007, p. 220-231. 
une véritable science, c'est-à-dire comme l'étude de faits observables, Watson abandonne l'idée de l'étude des motifs qui appartiennent à un état psychique, selon lui inatteignable et il restreint son étude au comportement. Cette position radicale n'est pas sans provoquer de réaction. Prenant le contre-pied de Watson, Yerkes affirme l'existence d'une mentalité animale et insiste sur le besoin de reconnaître les liens entre cette dernière et celle de l'homme. ${ }^{2}$ En pratique, cela signifie que Yerkes initie un nouveau programme de recherche. Il décide de travailler avec des singes qu'il va placer dans des situations expérimentales où ils pourront mettre en évidence des capacités d'apprentissage particulières.

Son objectif est alors d'aller au-delà des études menées par le psychologue américain Edward Thorndike (1874-1949), qui, à la suite du psychologue britannique Conwy Lloyd Morgan (1852-1936), avait postulé que les animaux apprennent seulement par «essais et erreurs", récompense et punition. Les animaux que Thorndike utilisait, principalement des chiens, des chats et des poules, étaient rendus affamés et maintenus prisonniers à l'intérieur de cages à mécanismes d'ouverture compliqués, les fameuses "boîtes à problèmes». Ils devaient apprendre à ouvrir la boîte afin d'aller chercher une petite quantité de viande ou de poisson placée à l'extérieur de la boîte. En mesurant le temps nécessaire pour chaque animal pour ouvrir la porte et atteindre la nourriture, Thorndike avait été capable de traduire de manière quantitative ces activités "d'intelligence» en traçant point par point le temps mis pour chaque essai successif, créant ainsi une courbe par animal.

Après avoir travaillé avec des petits animaux, Thorndike s'était procuré des singes et leur avait imposé plusieurs tâches toujours avec des "boîtes à problèmes». Avec les singes, de nouveaux résultats apparurent: les courbes, au lieu de décroître doucement, montraient un processus de "soudaine acquisition", indicatif de la présence d'un raisonnement. Cependant, Thorndike avait rejeté l'idée que les singes puissent raisonner. À ses yeux, les courbes indiquaient seulement l'établissement

2 Burnham, J. C., «Yerkes, Robert Mearns», in C. C. Gillispie (dir.), Dictionary of Scientific Biography, vol. 14, New York, Charles Scribner's Sons, 1970, p. 549. 
rapide d'un processus de «stimulus-réponse» qui avait lieu dans le cerveau des singes. ${ }^{3}$

Yerkes cherche à se démarquer des conclusions quasi-mécanistes de Thorndike sur les capacités mentales des animaux. Persuadé que ces derniers n'apprennent pas seulement par «essais et erreurs», il commence à travailler avec des singes. C'est un défi, car de tels animaux, denrées chères et fragiles, sont difficiles à se procurer sur le territoire américain. C'est un de ses étudiants, le psychiatre Gilbert van Tassel Hamilton (1877-1944), qui va permettre à Yerkes de réaliser son projet. Yerkes se rend à Montecito, près de Santa Babara, en Californie, où son ancien étudiant, devenu le médecin privé d'un millionnaire philanthrope, non seulement reçoit des honoraires pour ses services en tant que médecin mais aussi, a le privilège de consacrer une partie de son temps à ses recherches en psychologie. ${ }^{4}$ Grâce à son généreux mécène, Hamilton a pu se faire construire un laboratoire de psychologie comparée, incluant une petite colonie d'une quinzaine de singes. ${ }^{5}$ Pendant six mois de l'année 1915, dans des conditions matérielles et humaines optimales, Yerkes va être absorbé par sa première recherche systématique et approfondie sur le comportement des singes.

\section{Démarche et objectifs de recherche de Yerkes}

Lorsqu'il arrive à Montecito, Yerkes se met à travailler avec «l'appareil à choix multiples» que Hamilton a amélioré de manière conséquente depuis son installation en Californie. L'instrument que va utiliser Yerkes est beaucoup plus imposant que l'appareil initialement conçu: les quatre portes ont fait place à neuf portes qui ferment des sous-compartiments dans lesquels l'animal peut être emprisonné en cas d'échec à un test expérimental. Dans la procédure suivie par Yerkes, le sujet expérimental doit percevoir une certaine relation entre les portes qui lui font face,

3 Ce paragraphe est basé sur Boakes, R., From Darwin to Behaviourism: Psychology and the Mind of Animals, Cambridge, Cambridge University Press, 1984, p. 70-71 et Radick, G. op. cit., p. 204-209.

4 Wozniak, R.H., "Gilbert Van Tassel Hamilton and An Introduction to Objective Psychopathology", in Wozniak, R. H., (dir.), Behaviourism: the early Years, London, Routledge/ Thoemmes Press, 1994, p. xvi.

5 Lettre de R. Yerkes à G. Hamilton, 8 avril 1913, extrait du Fonds Yerkes, Archives de l'Université Yale, Boîte 23, Fichier 421. 
autrement dit, il doit apprendre une discrimination basée sur une position. ${ }^{6}$ Par exemple, la règle est la suivante: pour un jeu de portes données, seule celle qui est à l'extrême droite est toujours correcte. Si le sujet ouvre la porte correcte, il reçoit une récompense. Dans le cas inverse, il est puni et reste enfermé dans le sous-compartiment pendant quelques minutes. ${ }^{7}$ Selon Yerkes, cela décourage les choix hâtifs, hasardeux et négligents. ${ }^{8}$

Durant son séjour californien, Yerkes travaille en particulier avec deux macaques rhésus, du nom de Sobke et Skirrl, et Julius, un orang-outan âgé de cinq ans. Après avoir soumis ces animaux à une série de quatre problèmes à choix multiples, des résultats concluants apparaissent. ${ }^{9}$ Les courbes d'apprentissage des deux macaques présentent des pentes faibles, tandis que celle de Julius décrit une chute rapide, ce qui montre son habileté à articuler des idées. Après une vérification avec le test d'empilement des boîtes, Yerkes conclut que, contrairement aux deux macaques, «le grand singe est capable de montrer plusieurs formes de comportement d'idéation $»^{10}$, soit, en d'autres termes, faire preuve d'un comportement intelligent.

\section{Yerkes au service du biopolitique}

Ces expériences sur l'intelligence animale ont permis à Yerkes de faire du laboratoire le lieu incontournable de la recherche en psychologie comparée. Les primates ont été ses meilleurs alliés dans cette entreprise. Yerkes est persuadé que les singes, grâce à leurs ressemblances tant fonctionnelles que structurelles avec l'homme, peuvent contribuer à la solution des problèmes posés chez l'homme et aboutir à l'amélioration

6 Yerkes, R., The Mental Life of Monkeys and Apes, New York, Henry Holt (reprinted with an introduction y G.M. Haslerud, Delmar \& New York, Scholars' Facsimiles \& Reprints, 1979), 1916, p. 10.

7 Ibid., p. 11.

8 Ibidem.

9 Yerkes, R., «Ideational Behavior of Monkeys and Apes», Proceeding of the National Academy of Sciences of the United States of America, vol. 2, $\mathrm{n}^{\circ} 11$, p. 640.

10 Ibid., p. 642. Traduction personnelle. 
de sa condition. ${ }^{11}$ Cette conviction s'inscrit dans un engagement plus large en faveur d'une biologie et d'une psychologie toutes puissantes ouvrant sur un contrôle quasi-absolu de la nature. Yerkes est prêt à offrir son expertise en psychologie pour contribuer à l'amélioration de l'esprit humain. Il déclare:

La plupart d'entre nous sommes [sic] profondément convaincus que l'avenir de l'humanité dépend, dans une très large mesure, du développement de diverses sciences biologiques et sociales. [...] Nous devons [...] nous efforcer d'améliorer sans cesse davantage nos méthodes de mensuration mentale, car, désormais, il n'y a aucune raison de mettre en doute l'importante tant pratique que théorique des études sur le comportement humain. Il nous faut mesurer avec compétence chaque forme, chaque aspect du comportement humain ayant une signification psychologique et sociologique. ${ }^{12}$

Les études californiennes sur l'esprit animal dont Yerkes expose les résultats devant l'Académie des sciences américaine en 1916 vont être un atout pour promouvoir son expertise en matière de quantification de l'intelligence. Mais plus encore, c'est le premier conflit mondial qui va donner à Yerkes les moyens de mettre en pratique son expertise psychologique.

En 1917, alors que le conflit fait rage, Yerkes pousse l'Association de psychologie américaine (American Psychological Association) dont il est le directeur, à contribuer à l'effort de guerre. Très rapidement, il est nommé président du Comité pour l'examen psychologique des recrues (Committee on the Psychological Examination of Recruits) par le Conseil national de recherche (National Research Council ou NRC)..$^{13}$ On lui donne alors la charge de mettre au point des tests psychologiques pour sélectionner le personnel de l'armée. Yerkes va participer à la création et la mise en place des tests Alpha et Bêta qui seront utilisés sur 1750000 hommes en vue d'identifier les recrues avec un niveau d'intelligence

11 Yerkes, R., The Mental Life of Monkeys and Apes, New York, Henry Holt (reprinted with an introduction y G.M. Haslerud, Delmar \& New York, Scholars' Facsimiles \& Reprints, 1979), 1916, p. 135.

12 Yerkes (1917) cité par Gould, S. J., La mal-mesure de l'homme, Paris, Odile Jacob, 1997, p. 230 (traduction J. Cabert et M. Blanc de l'édition américaine de 1981).

13 Le National Reseach Council (NRC) est un organe de l'Académie des sciences américaine, créé en 1916 pour répondre à la demande croissante d'une expertise scientifique et technique pour supporter le premier conflit mondial. 
faible et, inversement, de permettre à l'armée de reconnaître les hommes qui seront aptes pour des entraînements spéciaux, comme les officiers. ${ }^{14}$ Ces tests sont aussi appliqués à une population plus large. Ils montrent alors que l'âge intellectuel moyen des Américains n'est pas de seize ans, mais de treize ans. Immédiatement, les eugénistes américains imputent la faiblesse de ce chiffre à des mariages entre personnes faibles d'esprit (de surcroît souvent pauvres), ainsi qu'entre la «race blanche» et les populations noires ou immigrantes. Yerkes adhère à ces vues eugénistes. Il est en effet persuadé que l'humanité ne peut que bénéficier de croisements judicieux et intentionnels entre populations sélectionnées.

C'est certainement cette pensée qui le conduit, après la première guerre mondiale, à continuer de collaborer avec le NRC, cette fois-ci au sein du Comité chargé des problèmes scientifiques de l'immigration (Committee on Scientific Problems of Human Immigration). En 1923 est publiée une étude, A Study of American Intelligence, de Carl Brigham, un disciple de Yerkes. Il y est démontré premièrement la supériorité de la race nordique (Nordic Race), deuxièmement que l'immigration de populations non blanches (principalement noire, puis d'Europe de l'est et méridionale) a globalement appauvri l'intelligence de la population américaine. Yerkes approuve cette étude et affirme qu'il est de son devoir civique de mettre en garde ses concitoyens de «ne pas ignorer la menace de la détérioration de la race ou des relations évidentes entre l'immigration et le progrès et de la sécurité nationale. ${ }^{15}$

En 1924, la Maison des Représentants et le Sénat américain voteront, avec une majorité écrasante, la Restriction Immigration Act. La loi sera rapidement promulguée sous le gouvernement du président Calvin Coolidge (1872-1933) qui, déjà en tant que vice-président, avait déclaré publiquement que: «[l]'Amérique doit rester américaine. Les lois biologiques montrent que ... les Nordiques dégénèrent lorsqu’ils se

14 Ces tests comprennent trois types d'examens: l'examen Alpha qui inclut huit sortes de tests, exige principalement la faculté de lire l'anglais, l'examen Bêta inclut, quant à lui, sept sortes de tests, consistant en des dessins, des figures, etc... et dont les instructions sont données par pantomime; enfin, le troisième examen, basé sur un test individuel, repose principalement sur l'application du test Stanford-Binet ou de l'échelle Yerkes-Bridge de mesure de l'intelligence.

15 Yerkes, R., «Foreword", in Brigham, C. C., A Study of American Intelligence, Princeton, Princeton University Press, 1923, p. vii ( Traduction personnelle). 
mélangent à d'autres races. ${ }^{16}$ Très vite cette loi conduira à la stabilisation des quotas migratoires, ceci pénalisant fortement l'entrée des immigrants en provenance de l'Europe de l'est et du sud.

L'engagement de Yerkes en faveur d'une ingénierie sociale et sa conviction du rôle de la psychologie dans l'organisation scientifique de la société sont révélés, dans leur forme la plus achevée, dans un rapport sur son travail dans les laboratoires rédigé à la fin de la seconde guerre mondiale: «[n] ous avons jugé important», concluait-il,

de convertir l'animal en un sujet de recherche biologique presque idéal et qui puisse être aussi mis en pratique. Et, à cette intention a été associé le soubait qu'un éventuel succès puisse servir de démonstration efficace montrant la possibilité de re-créer l'homme lui-même, à l'image d'un idéal généralement accepté. $^{17}$

Ainsi, Yerkes permit à la psychologie comparée d'acquérir une reconnaissance institutionnelle. Avec lui, l'intelligence animale devint un objet légitime de la psychologie, marquée au passage par le sceau de l'eugénisme américain. Fort de cette reconnaissance, Yerkes passa la majeure partie de sa carrière à étudier la vie psychologique et sociale des singes. En 1929, il publiera avec sa femme un ouvrage magistral pour la primatologie: The Great Apes. Dans cet ouvrage, Yerkes mentionne le nom d'un zoologiste français, Louis Boutan, qu'il cite comme précurseur de ses propres études sur le comportement d'idéation chez les singes: «il semble que Boutan ait découvert de ses observations sur le gibbon ce que j'ai révélé dans [mon] travail avec un orang-outan. ${ }^{18} \mathrm{C}^{\prime}$ est ce travail que nous allons étudier maintenant.

16 Voir Kevles, D.J., Au nom de l'eugénisme: génétique et politique dans le monde anglo-saxon, Paris, PUF, 1995, p. 139 (Traduction de M. Blanc de l'édition américaine de 1981).

17 Yerkes, R., The Chimpanzee: A Laboratory Colony (3rd ed.), New Haven, Yale University Press, 1945, p. 10 (Traduction personnelle).

18 Yerkes, R. et Yerkes, A., The Great Apes: A Study of Anthropoid Life, New Haven, Yale University Press, 1929, p. 184 (Traduction personnelle). 


\section{Des singes et des enfants: les études comparées sur l'intelligence animale de Louis Boutan}

\section{Quelques éléments biographiques sur Louis Boutan}

Si l'on cherche aujourd'hui sur internet des renseignements relatifs à Louis Boutan (1859-1934), on apprend qu'il fut l'un des pionniers de la photographie sous-marine davantage qu'un zoologiste qui aurait contribué au développement de la primatologie.

Boutan est né en 1859, à Versailles, dans une famille aisée et cultivée. Fort d'un double baccalauréat en sciences et en lettres, il commence des études de sciences naturelles à la faculté des sciences de Paris, à une époque où la zoologie expérimentale est en plein essor. La thèse de doctorat que Boutan soutient en 1886, porte notamment sur l'anatomie et le développement d'un mollusque marin, la fissurelle (Fissurella alternata $)^{19}$. En 1888, Boutan est nommé maître de conférences en zoologie à la faculté des sciences de Lille puis, quatre ans plus tard, à la faculté des sciences de Paris.

Tout en poursuivant sa carrière scientifique, Boutan nourrit sa passion pour les voyages et la mer. En 1880, il s'embarque pour l'Australie pour étudier l'embryologie des marsupiaux. ${ }^{20}$ Deux ans plus tard, il reçoit un financement pour organiser une expédition scientifique en Mer Rouge. Son goût pour la recherche scientifique à l'étranger est comblé lorsqu'en 1904, l'Académie des sciences de Paris lui offre de diriger une mission scientifique en Indochine, une mission qui durera quatre ans.

En janvier 1907, durant un court séjour dans le nord du Laos, Boutan acquiert un très jeune gibbon femelle aux joues blanches (Hylobates leucogenys), ${ }^{21}$ qu'il baptisera du nom de "Pépée». Ces petits singes agiles et arboricoles, armés de longs bras puissants et capables d'émettre des hululements, avaient toujours été une attraction des forêts humides du sud-est de l'Asie, dont ils étaient originaires. Comme les peuples locaux

19 Boutan, L., «Recherches sur l'anatomie et le développement de la Fissurelle. Comparaison de la Fissurelle avec les types voisins". Archives de Zoologie expérimentale et générale, $2^{\mathrm{e}}$ série, t. III bis, Supplément, 175 p., $14 \mathrm{pl}$.

20 Archives nationales, Archives Louis Boutan, F/17/24047: Dossier de carrière.

21 Boutan, L., «Le pseudo-langage: observations effectuées sur un anthropoïde, le gibbon (Hylobates Leucogenys - Ogilby) ", Actes de la Société Linnéenne de Bordeaux, 1913, vol. 67, p. 43. 
qui avaient intégré cet animal dans leur folklore, Boutan est fasciné par la capacité du gibbon à aller d'un arbre à un autre, par sa dextérité à se tenir debout et par ses chants mélodieux. Afin de trouver l'origine de développement de ses cris, Boutan l'élève de manière isolée dans son domicile à Hanoi. De retour en France, en 1908, Boutan poursuivra ses études sur l'esprit du gibbon, en s'intéressant cette fois-ci à ses capacités intellectuelles et à leur éventuel lien avec le développement du langage. Ainsi, le sujet de recherche de Boutan s'inscrit dans le débat autour de la question de l'évolution de l'esprit, débat qui n'a pas été sans soulever des polémiques en France au début du XXe siècle.

\section{L'étude de l'esprit animal dans un contexte post-darwinien}

Au tournant du $\mathrm{XX}^{\mathrm{e}}$ siècle, en France, l'affirmation darwinienne que l'homme et l'animal sont différents, non par nature mais par degrés, avait départagé la communauté scientifique en deux clans. D'un côté, se trouvaient les partisans de George Romanes (1848-1894), le disciple fidèle de Darwin qui, à sa demande, avait entrepris des études approfondies sur l'évolution de l'esprit. Parmi eux, on pouvait compter le biologiste Edmond Perrier (1844-1921), auteur en 1887 de la préface du livre de Romanes, Animal Intelligence (1882). Romanes avait adopté une position extrême en faveur de l'intelligence animale. Par exemple, dans Mental Evolution in Animals (1886) il allait jusqu'à dire qu' «il n'y avait pas de différence de nature entre l'acte de raison d'un crabe et celui d'un homme. ${ }^{22}$ Aux yeux de Romanes, il était clair que les animaux dépourvus de parole n'étaient pas dépourvus d'intelligence, et que la capacité pour le langage n'était qu'une étape supérieure dans le processus d'idéation. Emboîtant le pas à Romanes, Perrier soutenait l'idée que:

beaucoup d'animaux retiennent, abstraient, combinent leurs sensations, leurs souvenirs et leurs idées, acquièrent un véritable savoir, de la prévoyance et agissent fréquemment de manière quion ne puisse douter quils ont parfaitement saisi les relations des causes avec leurs effets [...] et se montrent véritablement intelligents. ${ }^{23}$

22 Romanes, G. (1883) cité par Richards, R., Darwin and the Emergence of the Evolutionary Theories of Mind and Behavior, Chicago and London, Chicago University Press, 1987, p. 347 (Traduction personnelle).

23 Perrier, E., «L'instinct», Bulletin de l'Institut général psychologique, 1900-1901, vol.1, p. 307. 
De l'autre côté, le biologiste Delage, mentor de Boutan, affirmait que:

faire dépendre les actes de l'animal d'opérations intellectuelles semblables à celles de l'homme n'est pas seulement une exagération, c'est une erreur radicale, car la pensée de l'animal ne differe pas de celle de l'homme seulement d'une façon qualitative, comme celle d'un illettré par rapport à celle d'un philosophe; elle en differe qualitativement, elle est d'une autre nature, et c'est là un point essentiel qui mérite d'être apprécié. ${ }^{24}$

Au début de son article de 1913, qui décrit les expériences linguistiques avec la gibbonne Pépée, Boutan emboîte le pas à Delage, en affirmant que les sons formulés par les singes ne "[peuvent] s'élever à la dignité des mots" et en posant l'existence d'un "pseudo-langage» inné et émotionnel. ${ }^{25}$ En premier lieu donc, Boutan avance des éléments qui accentuent le fossé entre l'animal et l'homme. Pour lui, il existe deux types de langages: un langage émotionnel que l'homme partage avec les animaux et un langage rationnel qui est spécifique à l'homme. Cependant, l'étude des oiseaux parleurs, notamment d'un merle buffle qu'il a capturé lors de son excursion au Laos, va remettre en cause cette affirmation première.

De retour à Hanoi avec l'animal capturé, Boutan découvre que le merle est capable d'apprendre les sons émis par la gibbonne Pépée. Pour Boutan, cela indique qu'il est capable d'un langage rudimentaire, comme le sont les oiseaux parleurs en général. Il dénomme ce langage le «stade merle buffle». ${ }^{26} \mathrm{Ce}$ «langage rudimentaire» lui apparaît comme très important en termes évolutifs puisqu'il met en évidence un état intermédiaire entre le "pseudo-language» et le langage humain, et par la même, remet en cause l'idée d'une barrière du langage.

En acceptant cette notion intermédiaire de "langage rudimentaire», Boutan semble donc avoir abandonné l'idée que le langage humain échappe à toute explication évolutionniste. Par la même on peut imaginer qu'il soit incliné à accorder une "raison rudimentaire» aux animaux. Ce tournant vers une pensée évolutionniste est renforcé

24 Delage, Y., "Comment pensent les bêtes», Bulletin de l'Institut général psychologique, 1911, vol. 11, p. 36.

25 Boutan, L. «Le pseudo-langage...», op.cit., p. 63.

26 Ibid, p. 71. 
lorsque, de retour en métropole, il entreprend ses études comparées avec sa gibbonne et des enfants.

\section{Analogies actives entre singes et enfants}

Le fait que Boutan ait pu relier le «stade merle buffle» avec celui où un enfant apprend à parler et commence à prononcer les mots de "maman" et "papa ${ }^{27}$, montre à quel point il pouvait admettre que les animaux et les enfants étaient placés sur le même continuum. Cette idée avait été renforcée à la fin du XIX ${ }^{e}$ siècle par les théories évolutionnistes, spécialement par celles d'un disciple et porte-parole de Darwin, Ernst Haeckel (1834-1919). Là où Darwin voyait des ressemblances entre des embryons comme la preuve d'une filiation commune, Haeckel prétendait que les formes inférieures de développement pouvaient être les équivalentes de stades premiers de développement d'espèces plus avancées. En 1866, Haeckel avait postulé la théorie de la récapitulation, à savoir, l'affirmation que l'ontogénie (c'est-à-dire le développement d'un individu) récapitule la phylogénie (c'est-à-dire l'histoire évolutive d'une espèce). Le livre monument de Haeckel, Natürliche Schöpfungsgeschichte (1868) avait été traduit en français en 1874 et, parmi les lecteurs attentifs de Haeckel, figurait le philosophe Hippolyte Taine (1828-1893), qui avait appliqué sa «Loi biogénétique fondamentale» à l'étude de l'esprit de l'enfant. Boutan s'inscrit dans le prolongement des travaux de Taine, tout en s'aventurant un peu plus loin: comme incarnation des degrés inférieurs de l'intelligence, l'enfant se trouve lui-même proche de l'animal, qui, de manière similaire, peut devenir un objet d'investigation. C'est cette pensée qui le conduit à entreprendre des études comparées sur les capacités mentales de sa gibbonne asiatique et celle d'enfants, âgés de deux à dix ans, c'est-à-dire, en possession ou non du langage. ${ }^{28}$ Surgissent alors différentes questions: dans quelle mesure un être humain privé de langage est-il capable de raisonner? Est-ce qu'un singe est capable de raisonner même s'il ne possède pas de langage? C'est de retour d'Indochine, en 1908, alors nommé professeur à l'université de

27 Ibid, p. 70-71.

28 Boutan, L., "Les deux méthodes de l'enfant", Actes de la Société Linnéenne de Bordeaux, 1914, vol. 68, p. 294. 
Bordeaux, que Boutan va conduire une série d'expériences en laboratoire avec Pépée et des jeunes enfants pour répondre à ces questions.

\section{Piéger le comportement spontané des singes et des enfants dans le laboratoire}

Le laboratoire de Boutan consistait en différentes pièces parmi lesquelles la "chambre d'observation" jouait un rôle central. Cette dernière communiquait avec la "chambre du gibbon", où la gibbonne devait manipuler des boîtes expérimentales sur une table et la "chambre des enfants», dans laquelle la même boîte que celle utilisée avec la gibbonne avait été posée directement sur le sol.

Réinvestissant ses talents de photographe, Boutan avait réalisé de nombreux clichés de la gibbonne et des enfants afin de justifier de la spontanéité de leur comportement. Dans ce but, la cloison entre la «chambre d'observation" et la «chambre du gibbon" avait été percée de deux ouvertures: l'une fermée par un verre coloré similaire à celui de la lampe d'une caméra photographique, l'autre fermée par un obturateur qui pouvait permettre de prendre des photos. À chaque moment, Pépée pouvait être observée par l'observateur sans qu'elle ne s'en rende compte.

Des conditions d'observation similaires avaient été prises avec les enfants. En laissant entrebâillée la porte de leur chambre de travail, Boutan pouvait regarder les faits et gestes des enfants à leur insu. ${ }^{29}$ Avant le début de chaque session, Boutan se cachait prudemment dans la "chambre d'observation" et attendait patiemment que l'enfant rentre dans la "chambre de travail» et se mette à manipuler la boîte à problème. Quand il jugeait que la session était finie, Boutan n'avait qu'à presser un bouton pour en informer son assistant. Ainsi, pour Boutan, le fait que la gibbonne/ l'enfant ne se sentent pas observés, assure qu'ils évoluent en toute liberté et expriment des comportements spontanés.

L'insistance de Boutan à observer un comportement spontané chez ses sujets expérimentaux est aussi révélée par la manière dont il conceptualisa son travail en termes intellectuels. Boutan est influencé par les idées d'éducation développées dans l'Emile, ou De l'éducation (1762) et qui sont valorisées, à la fin du XIX ${ }^{e}$ siècle par les réformateurs

29 Ibid., p. 295. 
de l'éducation, tels qu'Henri Marion, Pauline Kergomard, ou encore Ferdinand Buisson. Ces derniers défendent ce qu'ils appellent les "méthodes actives», méthodes qui visent à éveiller les facultés latentes de l'enfant, ses initiatives et à privilégier l'apprentissage plutôt que la réception passive de l'enseignement, et qui, comme Buisson l'indique dans son Dictionnaire de pédagogie et d'instruction primaire (1887) «ont pu tirer un excellent parti des observations de Rousseau. $»^{30}$

Comme Rousseau qui exigeait une relation étroite entre le tuteur et son élève, Boutan bénéficiait d'une grande familiarité avec son gibbon et les différents enfants avec lesquels il réalisa ses expériences. Le petit René, par exemple, dont il avait suivi le développement depuis l'âge de deux ans, était le fils aîné de son assistant de laboratoire. Selon Boutan, le jeune garçon " [avait été] élevé par de bons parents, qui l'[avaient] laissé se développer librement, sans contrainte et sans sévérité. $»^{31}$

Comme Rousseau qui avait fait du plaisir et du désir les moyens d'attirer l'attention d'un enfant, Boutan usait de l'appétit pour une nourriture attractive (aliment ou jouet selon qu'il s'agissait d'un enfant ou du gibbon) pour capter l'attention de ses sujets d'expérience. En fait, les boîtes expérimentales utilisées étaient recouvertes de grillage de telle sorte que l'aliment/le jouet puisse être directement perçu par la gibbonne/ l'enfant et ainsi stimulé leur désir. Aussi, Boutan avait pour principe de ne jamais forcer la gibbonne ou les enfants à aller au-delà de leurs limites fussent-elles psychologiques ou physiques: la gibbonne comme les enfants passaient par des exercices préliminaires et avaient la possibilité de faire des pauses entre les sessions expérimentales. Boutan avait insisté de manière récurrente sur le fait qu'il n'avait jamais dressé le gibbon que ce soit en dehors des sessions expérimentales ou durant les sessions elles-mêmes.

30 Buisson, F. (éd.), Dictionnaire de pédagogie et d'instruction primaire, Paris, Hachette, 1882-1887, p. 2642.

31 Boutan, L., "Les deux méthodes...», op. cit., p. 297. 


\section{Gibbon, enfants et les boîtes à problèmes: adapter les méthodes de la psychologie expérimentale américaine}

Les travaux de Boutan sont non seulement influencés par des données culturelles françaises, mais aussi par des travaux étrangers de psychologie expérimentale, en particulier ceux de Thorndike que nous avons mentionnés plus haut. Comme Yerkes, Boutan va se distancier des résultats de Thorndike qui, selon lui, accordent «trop peu à la puissance mentale de l'animal $»^{32}$.

En effet, Boutan juge les situations expérimentales développées par Thorndike trop compliquées et préfère des situations plus simples mettant en jeu un problème élémentaire et dans lequel la conduite de l'animal n'a qu'une signification. Même distance avec les «boîtes à problèmes» de Thorndike auxquelles Boutan préférera à des boîtes plus simples, quand bien même elles peuvent apparaître puériles au lecteur. ${ }^{33}$ De plus, alors que les boîtes de Thorndike avaient été conçues de manière à mettre en évidence un comportement par "essais et erreurs", celles de Boutan visent à discriminer un tel comportement d'un comportement intentionnel. Deux types de boîtes sont ainsi employées: des boîtes à mécanisme visible qui présentent une targette que l'on peut ouvrir grâce à un bouton ou en déplaçant horizontalement deux ou trois barres, une autre qui dépend d'un mécanisme d'ouverture invisible que l'on pouvait activer par une poire.

Avec les boîtes du premier type, l'enfant/ l'animal peut facilement déployer un comportement "d'essais et erreurs", comme pousser le bouton au lieu de le tirer, ou encore pousser la targette dans une mauvaise direction. Ce qui est spécial, aux yeux de Boutan, dans la conception de la boîte à mécanisme caché c'est qu'elle permet d'identifier la capacité à articuler des idées (c'est-à-dire à raisonner). En effet, à ce stade, sera démontré l'habileté du sujet expérimental à connecter deux notions: "presser la poire» et "ouvrir la boîte pour obtenir de la nourriture/ jouet».

Boutan est persuadé qu'un enfant de trois ans, grâce à la maitrise des mots, est capable de connecter ces deux notions. La question reste entière pour un enfant qui ne sait pas encore parler et pour la gibbonne. 
Sans langage à leur disposition, seront-ils capables d'articuler des idées qui puissent leur permettre d'ouvrir la boîte et ainsi de montrer qu'ils possèdent des rudiments de raison?

\section{La prudence de Boutan à attribuer des facultés de raisonnement au gibbon}

Dans la première expérience avec la boîte à targette, Pépée se trouve confrontée à un mécanisme qu'elle ne sait pas faire fonctionner. Après plusieurs essais, elle semble avoir appris que, pour ouvrir la boîte, elle doit bouger la targette de manière horizontale et non verticale. Aux yeux de Boutan, la gibbonne a acquis une notion qui peut être formulée de la manière suivante: «[p]ousser la targette dans une direction définie ouvre la boîte et permet de prendre la nourriture», et qui, puisque Pépée n'a pas de mots à sa disposition, doit se traduire en une série d'images associées comme celle de la boîte, du clapet, du bouton, de la targette... ${ }^{34}$

En passant au second type de boîte, les conclusions de Boutan s'avèrent plus radicales. Il affirme que sa gibbonne est loin de montrer de simples mouvements provoqués par des perceptions et des impulsions, mais agit de manière délibérée et apparemment réfléchie, avec une intentionnalité. ${ }^{35}$ Aux yeux de Boutan, ceci montre que la gibbonne est capable de ce qu'il appelle un "raisonnement embryonnaire» et que ses mouvement sont orientés par une idée. ${ }^{36} \mathrm{Il}$ affirme dans un premier temps:

[...] l'association entre le mouvement de la targette, le balancement de la poire et l'ouverture de la boîte [...] une fois établie [...] se fixe brusquement et le graphique donnerait une ligne droite à partir de ce moment. Si la notion a besoin de quelques tâtonnements pour devenir notion définitive, elle se précise trop rapidement, trop brusquement pour quion puisse dire que l'animal a contracté une habitude. ${ }^{37}$

Les résultats de Boutan remettent donc en cause les explications quasi-mécanistes de Thorndike relativement à l'apprentissage soudain chez les singes. Même si Boutan l'avance de manière prudente, les

37 Ibid., p. 256-257. 
expériences avec Pépée, nous dit-il, ont montré que «l'on trouve, chez eux [les singes], les indices d'une vie psychologique supérieure. ${ }^{38}$

\section{Là où la frontière entre nature et culture semble s'effacer}

Tout au long de sa recherche, Boutan a abordé la question de la domestication de sa gibbonne en termes idiosyncratiques. Bien que la gibbonne ait été maintenue dans des conditions de "semi-captivité» et dans un environnement humain, Boutan continua d'identifier son comportement comme spontané, c'est-à-dire comme naturel. Dès le premier jour où il entre en possession de l'animal orphelin (sa mère a été tuée par des chasseurs), il encourage sa femme à le traiter comme un "poupon».39 Par ailleurs, durant toute la vie de Pépée dans un environnement humain, Boutan donna carte blanche à sa femme pour s'en occuper: il ne posa aucune objection à ce qu'elle la traite comme un jeune enfant, l'habille selon les exigences de la mode de l'époque, lui apprenne à se tenir sur une chaise, à manger avec une cuillère, à boire dans un verre... En retour, la gibbonne vouait à la femme de Boutan une affection exclusive, tant elle considérait cette mère adoptive comme "son défenseur naturel $»{ }^{40}$

Ainsi, alors que Boutan reconnaissait que Pépée avait été élevée dans un environnement domestique et avait acquis une éducation basique, au même moment, il croyait que, du fait qu'elle avait été gardée sans chaîne dans la maison (dans ce qu'il appelait des conditions de «semi-liberté»), elle avait exprimé un comportement spontané. Comment pouvait-il se bercer d'une telle illusion?

Il semble bien que dans le cadre intellectuel de Boutan, la frontière entre le naturel et l'artificiel apparaisse comme brouillée. Pourquoi cela? Clairement, Boutan avait affiché le souci de distinguer ses travaux de ceux des dresseurs d'animaux, qui, à ses yeux, en considérant l'animal comme une mécanique, avaient permis chez eux le déploiement de comportements artificiels. De la même manière, le comportement de sa gibbonne ne pouvait en rien être comparé à celui des animaux maintenus en stricte captivité, dans les zoos ou dans les cirques. Par ailleurs, on

38 Ibid., p. 265.

39 Boutan, L., "Le pseudo-langage... », op. cit., p. 44, note de bas de page (1). 40 Ibid., p. 54. 
peut supposer que la définition de la nature par Boutan avait pu évoluer au cours de ses nombreux séjours à l'étranger, et particulièrement après son expérience dans les colonies françaises d'Asie puis, à la fin de sa vie, d'Afrique du nord. Des photographies prises dans un contexte privé révèlent combien Boutan appréciait s'imprégner des cultures découvertes au cours de ses voyages, au point de changer ses habitudes ou de modifier son cadre de vie. Ainsi, un cliché pris en Algérie le voit dans une tenue bédouine, la tête enrubannée, tout en étant plongé dans la lecture d'un livre et confortablement installé dans un fauteuil richement décoré du style Napoléon III.

Ainsi, Boutan semble avoir été imprégné par les cultures des pays, tout en conservant ses propres valeurs bourgeoises françaises. Plus qu'un transfert à sens unique de la métropole vers les colonies, on peut envisager davantage ici un échange entre cultures étrangères et métropolitaines. Tout comme Boutan à la fin de sa vie n'incarne plus tellement l'universitaire type de la Troisième République française, sa gibbonne Pépée, après un long séjour en France, ne se comporte plus comme ses congénères sauvage. Dans les deux cas, un processus d'acculturation a eu lieu qui aura, en partie, déplacé la frontière entre nature et culture. D'où l'illusion de Boutan à voir dans son gibbon un animal exhibant un comportement naturel.

En combinant des principes rousseauistes d'éducation, des conceptions évolutionnistes et des pratiques scientifiques de la psychologie expérimentale américaine, Boutan suggéra que les singes étaient intelligents. Si le travail de Boutan en psychologie animale fut jugé comme précurseur par Yerkes, il ne fut jamais reconnu à sa juste valeur par ses pairs français. Par exemple, le psychologue Paul Guillaume (1878-1962) n'accorda pas beaucoup de valeur aux recherches de Boutan et les considéra davantage comme un travail d'amateurs. ${ }^{41}$ Ce sont les études de Guillaume sur les singes, menées en collaboration avec Ignace Meyerson (1888-1983) que nous allons étudier maintenant.

41 Guillaume, P., La psychologie animale, Paris, Armand Colin, 1940, p. 192-194 \& 1941, p. 262 \& p. 324-328. 


\section{Guillaume et Meyerson : l'étude expérimentale de l'intelligence animale}

L'année 1927 marque le début d'une longue collaboration professionnelle et amicale entre les psychologues Guillaume et Meyerson. ${ }^{42}$ Les deux hommes ont une formation similaire, à la fois en philosophie et en sciences naturelles. Agrégé de philosophie, Guillaume est aussi titulaire d'un diplôme de physiologie et d'embryologie. Ses intérêts de recherche tournent autour de la psychologie de l'enfant et de l'animal. En 1925, Guillaume a soutenu une thèse sur l'imitation chez les enfants et, en 1927, traduit le livre de psychologie gestaltiste Wolfgang Köhler sur l'intelligence des singes. Aux yeux de Guillaume, psychologie de l'enfant et psychologie animale sont complémentaires: «l'on pourrait tester les résultats obtenus avec des enfants avec le travail actuel de psychologie animale. $»^{43}$ En effet, pour Guillaume, les expériences avec les enfants ne sont pas fiables: "dès qu'un enfant ou un homme sent qu'on veut savoir quelque chose sur son caractère, il ment, il est réticent, il pose, il cherche à nous imposer une certaine idée de lui-même, il fabule; on ne le voit pas tel qu'il serait s'il n'avait pas cette préoccupation de se déguiser $»^{44}$. Au contraire de l'enfant qui «se dérobe», l'animal est comme un «objet physique» qui «ne se défend pas contre la curiosité de l'expérimentateur ». ${ }^{45}$ Par ailleurs nous dit Guillaume, les expériences sur les animaux ne sont pas entravées par des obstacles éthiques, et des expériences comme la destruction de zones cérébrales sont possibles, alors qu'elles ne le seraient pas sur des humains. ${ }^{46}$ Ainsi, pour Guillaume, la psychologie animale offre des possibilités expérimentales plus larges que la psychologie de l'enfant.

Meyerson, quant à lui, est né en Pologne et a émigré en France avec sa famille en 1906. Très influencé par son oncle, le philosophe Émile Meyerson (1859-1933) qu'il retrouve à Paris, Meyerson prépare une

42 Meyerson, I., «Paul Guillaume», Journal de Psychologie normale et pathologique, 1962, vol. LIX, p. 1.

43 Guillaume, P., L'imitation chez l'enfant, Paris, PUF, 1950, p. 207.

44 Archives nationales (Centre des archives contemporaines), Archives Ignace Meyerson, $521 \mathrm{AP} 18$ : Centre de synthèse, $13^{\mathrm{e}}$ semaine de synthèse, procèsverbal dactylographié de conférences et de discussions: "La psychologie animale», par Paul Guillaume, 28 mai 1947, p. 23.

45 Ibidem.

46 Ibidem. 
licence de sciences naturelles et entreprend des études de médecine entre 1907 et 1914, tout en suivant des cours de philosophie (il obtiendra sa licence en 1918). En 1928, il est chargé de conférences et d'un cours de psychologie à La Sorbonne. C'est aussi à cette époque qu'il commence son travail sur la psychologie des singes avec Guillaume. En 1930, ils présentent une communication à la Société de psychologie sur «L'usage de l'instrument chez les singes» et projettent un film qui rend compte de leurs travaux. Les résultats de ces études seront publiés dans le Journal de psychologie normale et pathologique entre 1930 à 1937 et repris dans un volume, publié chez Vrin en 1937, sous le titre: Recherches sur l'usage de l'instrument chez les singes.

C'est à la demande du psychologue français Georges Dumas (et aussi avec l'appui du médecin Albert Calmette) que Guillaume et Meyerson vont étudier la psychologie des singes. Ils mèneront leurs travaux entre 1927 et 1938 à la ménagerie du Museum national d'histoire naturelle et à l'Institut Pasteur de Paris. Ces lieux leur sont imposés et Guillaume n'a de cesse de se plaindre qu'en France, au contraire des États-Unis, de l'Allemagne ou de la Russie, «il n'existe pas de laboratoires de recherche spécialement destinés à l'étude des singes. $»^{47}$ Guillaume aussi se lamente du manque d'animaux sains et adaptés à de telles recherches. En effet, les singes dont il hérite sont passés entre les mains de médecins de l'Institut Pasteur et ont subi de nombreuses expérimentations médicales. Notamment, ils ont pu servir de cobayes pour tester des vaccins contre la tuberculose.

De manière similaire, Meyerson envie ceux qui, comme Yerkes, ont "réalisé le rêve qu'il caress[ait] depuis longtemps, [...] d'aller à Kindia», un Institut Pasteur d'Outre-Mer situé en proximité de forêts peuplées de singes, en Guinée française. C'est aux yeux de Meyerson, «évidemment le seul moyen de voir les singes tels qu'ils sont vraiment" et de "travailler dans des conditions de [son] rêve». À cet idéal africain, Meyerson oppose son cadre quotidien de travail, en métropole. C'est à Yerkes encore, au détour d'une lettre, que Meyerson n'hésite pas à faire part de ses déceptions: «depuis deux ans que dure mon travail, bien que

47 Guillaume, P., «Recherches sur l'usage de l'instrument chez les singes, I Le problème du détour", Journal de psychologie normale et pathologique, 1930, vol. XXVII, p. 178. Voir aussi Guillaume, P. La Psychologie animale, Paris, Armand Colin, 1940, p. 45. 
j'aie eu la chance d'avoir d'excellents sujets (notamment une chimpanzé [sic] remarquable), j'ai toujours eu l'impression que je travaillais dans de mauvaises conditions. $»^{48}$

\section{Guillaume et Meyerson reproduisent les découvertes de Köhler sur l'insight}

Meyerson et Guillaume travailleront pendant dix ans sur une trentaine de singes appartenant à treize espèces différentes, en particulier des chimpanzés, parmi lesquels ils privilégieront sept animaux. De manière répétée, ils les soumettront à différents exercices de difficultés croissantes, s'inspirant largement des travaux sur les singes menés par Köhler entre 1914 et 1924, à Ténérife, dans les îles Canaries et qui mirent en évidence leur capacité d'insight, ou, dit plus simplement, leur intelligence. ${ }^{49}$

Köhler était convaincu que les performances d'un animal ne pouvaient s'expliquer uniquement par un apprentissage par "essais et erreurs" et, à l'instar de Yerkes et de Boutan, il imagina des situations expérimentales suffisamment simples pour que l'animal puisse comprendre des problèmes élémentaires, dans lesquels sa conduite n'ait qu'une seule signification. Parmi ces situations, les tests du détour illustrent au mieux le travail de Köhler.

Dans de tels tests, l'animal est placé dans une situation où il est à proximité d'un objet qu'il désire (le plus souvent de la nourriture), mais dont l'accès lui est interdit par un obstacle, un filet, ou des barres. Pour atteindre l'objectif, l'animal doit contourner l'obstacle. La situation est délicate car, à un moment du détour, l'animal perd de vue l'objet désiré. Il y trois niveaux de tests: a) l'animal commence par faire un angle de 90 degrés en direction de l'objectif, qui reste dans son champ visuel, b) l'animal doit faire un détour qui implique un angle de 125 degrés, c) le détour conduit l'animal dans une direction diamétralement opposée à

48 Lettre d'Ignace Meyerson à Robert Mearns Yerkes, Paris, datée du 17 juin 1929, extrait du Fonds Robert Yerkes, Archives de l'Université Yale, Boîte 34, Fichier 650.

49 Guillaume, P., «Recherches sur l'usage de l'instrument chez les singes, I Le problème du détour ", op. cit., p. 178-181. 
l'objet convoité et où ce dernier disparaît de la vue de l'animal sur une longue distance.

Pour Köhler, ce dernier test, le plus difficile, révèle qu'un animal est doué d'insight, soit un processus mental qui accompagne une reconnaissance soudaine de la solution à un problème. Cette reconnaissance marquée par un changement brutal dans la conduite de l'animal se produit car il a vu la situation d'une autre manière. Selon Köhler, un processus de "restructuration perceptuelle» s'est produit, ${ }^{50}$ processus qui se traduit graphiquement par «l'interruption de la courbe continue essais/ temps en une chute rapide. ${ }^{51}$ Il est aussi important de noter que, pour un psychologue de la Gestalt, l'on ne doit pas assumer a priori que le monde perçu par un animal est similaire au nôtre, mais l'on doit plutôt trouver des expériences adaptées qui vont pouvoir mettre en évidence la manière avec laquelle le monde apparaît à l'animal que l'on étudie. ${ }^{52}$

Dans les expériences de détour de Guillaume et Meyerson, la situation est différente de celle imaginée par Köhler, mais comme chez Köhler, le point de vue de l'animal est central. Comme l'écrit Guillaume: «l'expérimentateur crée une situation où lui-même aperçoit ces diverses possibilités, mais il n'est pas toujours sûr que l'animal les ait toutes lui-même. ${ }^{53}$ À l'inverse des expériences de Köhler où les

50 Extrait de Boakes, R., From Darwin to Behaviourism, op. cit., p. 191.

51 Ash, M. G., Gestalt Psychology in German Culture, 1890-1967: Holism and the Quest for Objectivity, Cambridge, Cambridge University Press, 1995, p. 157.

52 Prenons le cas où un singe doit faire passer une échelle à travers des barres de fer. Pour l'homme, le problème est très simple: il sait qu'il doit qu'il doit maintenir l'échelle parallèlement aux barres de fer afin qu'elle puisse passer à travers. Le singe pour sa part, essaie de la faire passer en la prenant dans n'importe quel sens. Il n'a pas une vision claire de la perception de l'ensemble échelle-barre de fer. En fait, le singe fait face à un problème similaire de celui d'un homme qui doit démêler un écheveau de coton. Il essaie de le défaire, prudemment, et de le réorganiser en unités séparées. Extrait des Archives nationales (Centre des archives contemporaines), Archives Ignace Meyerson, $521 \mathrm{AP}$ 18: Centre de synthèse, $13^{\mathrm{e}}$ semaine de synthèse, procès-verbal dactylographié de conférences et de discussions: "La psychologie animale», par Paul Guillaume, 28 mai 1947, p. 39.

53 Guillaume, P., «Recherches sur l'usage de l'instrument chez les singes, IV Choix, Correction, Invention", Journal de psychologie normale et pathologique, 1937, vol XXXIV, p. 425. 
animaux évoluent dans un enclos, soit en semi-captivité, les chimpanzés avec lesquels travaillent Guillaume et Meyerson sont maintenus dans des cages et leurs gestes sont entravés par des barreaux. Par exemple, ils manipulent un bâton afin d'atteindre une banane qui a été déposée sur une écritoire, à l'extérieur de leur cage, alors que les singes de Köhler tentaient d'attraper une banane accrochée au plafond d'un enclos à l'aide de boîtes empilées. Guillaume et Meyerson sont donc contraints de procéder à des modifications par rapport aux expériences de Köhler. Ce n'est pas l'animal qui se déplace vers l'objet de convoitise, mais cet objet qui pourra être déplacé vers l'animal. Dans les tests préliminaires avec un bâton (équivalent aux tests a) et b)), une banane est déposée sur une écritoire en face du singe qui, à l'aide d'un bâton, doit l'attirer à lui pour la récupérer. Dans ce cas, le geste de l'animal est très simple et obéit à une certaine idée de la préhension: saisir quelque chose signifie l'approcher à soi. Mais, dans le test du détour avec la "caisse creuse" (qui se veut l'équivalent du test c)), la difficulté consiste à devoir pousser l'objet que l'animal souhaite atteindre dans une direction opposée, vers un espace où il n'y a rien. Pour Guillaume, cela prouve que l'animal est capable de se détacher de la situation actuelle, immédiate, concrète et de se poser, en quelque sorte, un but idéal. ${ }^{54}$ À ses yeux, cette expérience est très significative d'un changement dans la perception du monde par le singe: il est passé d'une perception auto-centrée à une perception plus objectivée de la réalité. Il a fait preuve d'intelligence.

\section{Guillaume et l'apologie du laboratoire}

À la fin des années 1920, lorsque Guillaume et Meyerson entreprennent leurs études sur les singes, la psychologie est sortie du giron de la philosophie et a assis sa méthodologie sur les prérogatives du laboratoire, à savoir, le contrôle, la répétition et la quantification. ${ }^{55}$ Si lors d'une conférence sur la psychologie animale, Guillaume se fait attaqué pour aborder expérimentalement des questions philosophiques, sa ripostene se fait pas attendre: «[j]e ne voudrais pas me laisser entraîner hors de mon terrain, qui était celui d'une science terre à terre,

54 Ibidem.

55 Guillaume, P., La psychologie animale, op. cit., p. 44. 
et non celui de la métaphysique. " ${ }^{56}$ Pour reprendre plus loin quand on continue à l'interroger sur la portée philosophique de son travail: «[m] on but était simplement de montrer que la psychologie animale nous place sur le terrain de l'expérience, et non de poser le problème général de la finalité. ${ }^{57}$

Aux yeux de Guillaume, conduire des expériences dans les conditions artificielles du laboratoire est la garantie de la scientificité de son travail. Le laboratoire, nous dit-il, est «un milieu choisi où l'on s'entoure de moyens de mieux voir, de mesurer, d'enregistrer» et qu'il «sera toujours avantageux de remplacer les conditions imposées par la nature par les conditions librement choisies du laboratoire, en y transportant l'animal, en l'y faisant vivre. ${ }^{58}$ L'insistance de Guillaume sur la valeur de l'expérimentation réalisée en laboratoire est aussi évidente dans la manière dont il décrit l'approche de terrain:

Le "field naturalist" aura peut-être la chance de trouver des anomalies significatives: un jour il trouvera un nid d'oiseau fait de matériaux insolites, par exemple de déchets industriels; il verra une mouche pondre sur une plante à odeur cadavérique et non plus sur un cadavre, une autre laisser tomber ses oufs sur une glace et non plus sur le miroir des eaux. Ces trouvailles jettent une lumière sur certaines conditions de la construction et de la ponte normale. Mais ces occasions sont bien rares et la méthode fait dépendre le progrès d'une série de hasards heureux. Le simple rapprochement des données de l'observation ne suffit donc pas pour trancher les questions sans le recours de l'expérimentation. 59

Ainsi, par opposition aux observations sur le terrain souvent hasardeuses, toujours fragmentaires et difficiles à réaliser, l'expérimentation en laboratoire permet de renseigner de manière sûre sur le déterminisme du comportement. Certes, si l'observateur de terrain possède des qualités d'endurance, de patience et la capacité à inventer des ruses pour approcher les animaux, ${ }^{60}$ ses travaux restent descriptifs et systématiques: «l'observation», nous dit Guillaume, "constate des comportements,

56 Archives nationales (Centre des archives contemporaines), Archives Ignace Meyerson, $521 \mathrm{AP} 18$ : Centre de synthèse, $13^{\mathrm{e}}$ semaine de synthèse, procèsverbal dactylographié de conférences et de discussions: "La psychologie animale", par Paul Guillaume, 28 mai 1947, p. 32.

57 Ibid., p. 33-34.

58 Guillaume, P., La Psychologie animale, op. cit., p. 44.

59 Ibid., p. 36-37 (en anglais et en italiques dans le texte original).

60 Ibid., p. 32-33. 
mais elle n'apprend rien sur leur déterminisme. Elle montre comment l'animal se conduit dans les conditions naturelles, mais elle ne peut pas séparer ce qui est vraiment condition de ce qui est n'est que simple circonstance, et risque d'attribuer à certaines concomitances une importance qu'elles n'ont pas. ${ }^{61}$

Cette mise en évidence des limites du terrain et la défense du laboratoire est forte au point de modifier sa perception du naturel:

Un singe est enfermé dans une cage; en dehors, hors de portée de sa main, on place un appât qui le tente, et on lui donne un instrument dont un homme pourrait se servir pour attirer l'appât. Cette situation ne se trouve pas dans le milieu naturel. La solution que l'animal inventera peut-être semble être ici autre chose que la reproduction, dans des conditions plus claires pour nous, d'un comportement naturel. Ce qui nous intéresse ici, c'est d'apprendre comment le singe va répondre à cette situation artificielle prise en elle-même. Peu importe quil n'ait jamais eu l'occasion de résoudre de tels problèmes. Mais son acte, pour être nouveau, n'en est pas moins réel. Il est naturel, en ce sens quill répond à des possibilités qui font partie de sa nature. Il s'agit de définir, pour toutes les situations possibles, ces virtualités dont ses actes ordinaires ne sont que des cas particuliers. ${ }^{62}$

Il conclut de manière assez radicale par une oblitération totale du caractère artificiel des études en laboratoire: "La distinction de situations naturelles et artificielles, de réactions naturelles et artificielles n'a donc pas de signification profonde; $[\ldots] »^{63}$.

\section{Conclusion}

Yerkes, en imposant les singes comme nouveaux animaux de laboratoire et en sollicitant de manière stratégique des financements, est considéré comme l'une des figures clés de la primatologie. Au-delà de ce destin scientifique brillant, l'examen des travaux de Yerkes a montré à quel point l'étude du comportement animal peut s'articuler avec la question du contrôle social. Au début du XXe siècle, l'intelligence animale puis humaine devint entre les mains des scientifiques et des

61 Archives nationales (Centre des archives contemporaines), Archives Ignace Meyerson, 521 AP 18: Centre de synthèse, $13^{e}$ semaine de synthèse, procèsverbal dactylographié de conférences et de discussions: "La psychologie animale», par Paul Guillaume, 28 mai 1947, p. 21.

62 Guillaume, P., La Psychologie animale, op. cit., p. 42-43.

63 Ibid., p. 43. 
politiques un objet puissant qui contribua à la normalisation du vivant. Les études françaises sur le comportement animal, quant à elles, illustrent une approche de la psychologie animale qui cultive un intérêt pour l'étude des phénomènes de conscience et qui se détache de la psychologie introspective, tout en montrant que l'esprit animal est le lieu d'une intelligence. Boutan, on l'a vu, aura une certaine réticence à donner l'intelligence aux singes, alors que Meyerson et Guillaume, en appliquant les théories de la Gestalt et en défendant une psychologie expérimentale, s'autoriseront à faire des singes des individus intelligents. Cependant, il y a des similarités entre le travail de Boutan et les études de Guillaume et Meyerson. Dans les deux cas, les études sur la psychologie des singes ont été conduites dans des territoires marginaux: Boutan, isolé de la communauté parisienne, se livra à ses études de psychologie comparée dans son laboratoire de province. Guillaume et Meyerson poursuivirent leurs études dans des territoires qui n'étaient pas dédiés à l'étude du comportement animal et travaillèrent avec des animaux de "seconde main" (utilisés en premier lieu pour la recherche biomédicale). Boutan, comme Guillaume et Meyerson, réalisèrent leurs études sans ambitieux programme scientifique: aucun d'entre eux ne chercha à faire de la psychologie des singes les bases d'une primatologie de plus grande envergure (comme ce sera le cas pour l'Américain Yerkes). Cela peut expliquer pourquoi, Boutan, comme Guillaume et Meyerson, sont des personnalités scientifiques qui figurent rarement dans les récits de primatologie et que, globalement, l'histoire de la primatologie française a été oubliée. 